\title{
Intramolecular dehydrogenative coupling of biaryl tertiary amines promoted with $t$-BuOK/DMF: A convenient synthesis of 6-aryl-5,6-dihydrophenanthridines
}

\author{
Ting Zhang, Lu Qian, Zhen-yu Chen, Yong Zou, Xue-jing Zhang,* and Ming Yan* \\ Institute of Drug Synthesis and Pharmaceutical Process, School of Pharmaceutical Sciences, \\ Sun Yat-sen University, Guangzhou 510006, China \\ Email:zhangxi33@mail.sysu.edu.cn
}

Received 12-24-2016

Accepted 01-22-2017

Published on line 05-14-2017

\section{Abstract}

A number of 6-aryl-5,6-dihydrophenanthridines were prepared in good yields via an intramolecular dehydrogenative coupling of biaryl tertiary amines promoted by $t$-BuOK/DMF. A reaction mechanism involving $\alpha$-aminoalkyl radical intermediates is suggested.
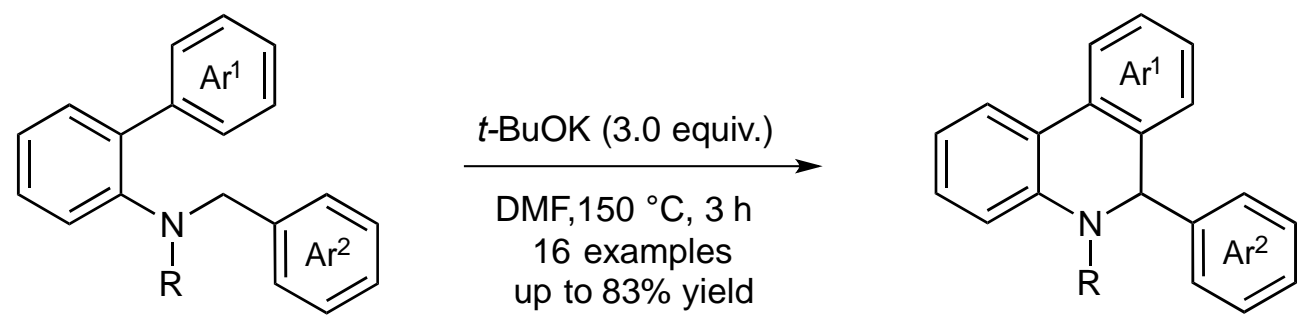

Keywords: Tertiary amine, $\alpha$-aminoalkyl radical, cross-dehydrogenative coupling, dihydrophenanthridine 


\section{Introduction}

Recently, the construction of carbon-carbon bonds via cross-dehydrogenative coupling (CDC) from two simple $\mathrm{C}-\mathrm{H}$ bonds has made great progress. ${ }^{1-10}$ This strategy, introduced by $\mathrm{Li}$ and other researchers, is demonstrated to be a superior alternative to classic coupling procedures using prefunctionalized starting materials. CDC reactions are generally achieved in the presence of transition-metal catalysts and sacrificial oxidants or $\mathrm{H}$ acceptors. In recent years, transition-metal-free CDC reactions have also been developed. ${ }^{11-16}$ In 2015 , Wu and co-workers developed a $t$-BuOLi-promoted CDC reaction of quinolone $N$-oxides and 1,3-azoles without external oxidants. ${ }^{17} \mathrm{~A} t$-BuOK/DMSO mediated intermolecular CDC reaction of nitroarenes and indoles in an open flask was also reported. ${ }^{18}$ These transformations usually proceed via oxidative SET (single electron transfer) $\mathrm{C}-\mathrm{H}$ activation and the subsequent generation of radical intermediates.

Recently, our group has developed a series of $t$-BuOK/DMF promoted coupling reactions of tertiary amines, amides and diphenylmethanes with alkenes, alkynes and ketones. ${ }^{19-25}$ The formation of $\alpha$-amino alkyl radicals or diphenylmethyl radicals initiated by $t$-BuOK/DMF was proposed. We speculated that $\alpha$-amino alkyl radicals would also react with arenes which could provide a new entry to the preparation of polycyclic heterocyclic compounds, such as 5,6-dihydrophenanthridine derivatives. Dihydrophenanthridine derivatives are present in the skeleton of a wide number of biologically active compounds, natural products and materials, ${ }^{26-29}$ however, only a few synthetic methods for this class of compounds have been reported. ${ }^{30-35}$ Furthermore, the reported methods generally suffer from limited functional group tolerance and undesired in situ oxidation. Herein, we report an intramolecular dehydrogenative coupling of biaryl tertiary amines promoted by $t$-BuOK/DMF. The reaction provides a new synthetic approach to 6-aryl-5,6-dihydrophenanthridines (Scheme 1).

\section{1) Previous work}<smiles>C=Cc1ccccc1N1CCc2ccccc2C1</smiles>

$$
\underset{\mathrm{DMF}, 90^{\circ} \mathrm{C}, 3 \mathrm{~h}}{\stackrel{\mathrm{BuOK}}{(1.5 \text { equiv. })}}
$$<smiles>c1ccc2c(c1)CCN1c3ccccc3CCC21</smiles><smiles>[R]C(=O)c1ccccc1N1CCc2ccccc2C1</smiles>

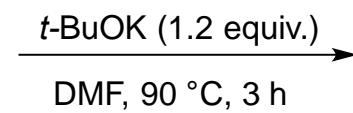<smiles>[R]c1c2n(c3ccccc13)CCc1ccccc1-2</smiles>

2) This work<smiles>CN(Cc1ccccc1)c1ccccc1-c1ccccc1</smiles>

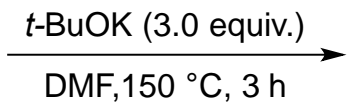<smiles>CN1c2ccccc2-c2ccccc2C1c1ccccc1</smiles>

Scheme 1. $t$-BuOK /DMF promoted carbon-carbon bond formations. 


\section{Results and Discussion}

Initially, we examined the reaction of $1 \mathrm{a}$ in DMF with 3.0 equiv. of $t$-BuOK at $120{ }^{\circ} \mathrm{C}$. To our delight, $2 \mathrm{a}$ was obtained in a moderate yield. Furthermore, a number of bases and reaction solvents were examined and the results are summarized in Table 1. $t$-BuONa, $t$-BuOLi, and $\mathrm{K}_{2} \mathrm{CO}_{3}$ were tested, however, no $2 \mathrm{a}$ was obtained (Table 1, entries 2-4). The reaction in DMSO was also applicable, but a lower yield was observed (Table 1, entry 5). THF was found to be incompatible with the reaction and no product $2 \mathrm{a}$ was obtained. The effect of $t$-BuOK loading was also examined and the best yield was obtained with 3.0 equiv. of $t$-BuOK (Table 1, entries 7-8). The reaction was tested at 90,150 and $180{ }^{\circ} \mathrm{C}$ respectively. The reaction at $150{ }^{\circ} \mathrm{C}$ gave the best result (Table 1, entries 9-11).

Table 1. Optimization of reaction conditions ${ }^{a}$

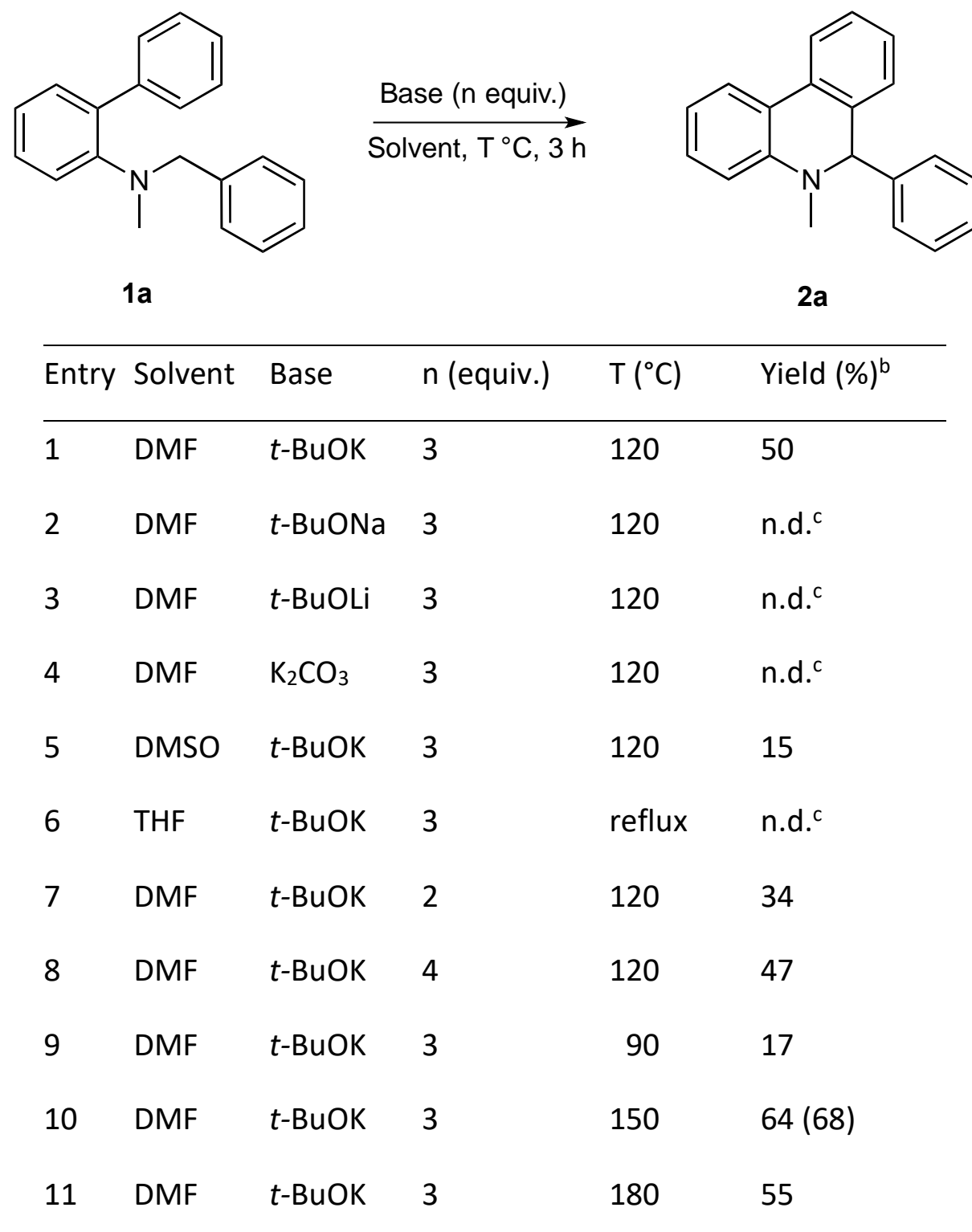

${ }^{a}$ Reaction conditions: 1 a $(0.2 \mathrm{mmol}, 1.0$ equiv.), base ( $\mathrm{n}$ equiv.), solvent $(2.0 \mathrm{~mL})$, at the indicated temperature for 3 hours under nitrogen atmosphere. ${ }^{b}$ Yields were obtained by GC with $n$-dodecane as the internal standard. The value in the parentheses is the isolated yield after column chromatography. ${ }^{c}$ Not detected. 
<smiles>[R]N(Cc1ccccc1)c1ccccc1-c1ccccc1</smiles>

1a-q
t-BuOK (3.0 equiv.)

DMF, $150{ }^{\circ} \mathrm{C}, 3 \mathrm{~h}$

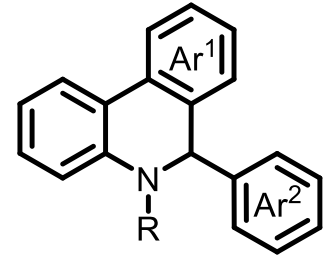

2a-q<smiles>CN1c2ccccc2-c2ccccc2C1c1ccccc1</smiles>

2a, $68 \%$<smiles>COc1ccc(OC)c(C2c3ccccc3-c3ccccc3N2C)c1</smiles><smiles>CN1c2ccccc2-c2ccccc2C1c1ccc(C(F)(F)F)cc1</smiles>

$2 \mathbf{i}$, n.d.<smiles>COc1ccc(C2c3ccccc3-c3ccccc3N2C)cc1</smiles>

2b, $83 \%$<smiles>Cc1ccc(C2c3ccccc3-c3ccccc3N2C)cc1</smiles>

2f, $64 \%$<smiles>CN1c2ccccc2-c2ccccc2C1c1ccc2ccccc2c1</smiles>

2j, $70 \%$<smiles>COc1cccc(C2c3ccccc3-c3ccccc3N2C)c1</smiles>

2c, $82 \%$<smiles>Cc1cccc(C2c3ccccc3-c3ccccc3N2C)c1</smiles>

2g, $53 \%$<smiles>CN1c2ccccc2-c2ccccc2C1c1cccs1</smiles>

$2 k$, n.d.<smiles>COc1ccc(C2c3ccccc3-c3ccccc3N2C)cc1OC</smiles>

2d, $72 \%$<smiles>Cc1ccccc1C1c2ccccc2-c2ccccc2N1C</smiles>

2h, $35 \%$<smiles>CN1c2cccc3cccc(c23)C1c1ccccc1</smiles>

2I, n.d.<smiles>Cc1cccc2c1-c1ccccc1N(C)C2c1ccccc1</smiles>

2m, $41 \%$<smiles>Cc1ccc2c(c1)C(c1ccccc1)N(C)c1ccccc1-2</smiles>

2n, $27 \%$<smiles>CN1c2ccccc2-c2ccncc2C1c1ccccc1</smiles>

$20,68 \%$<smiles>CCN1c2ccccc2-c2ccccc2C1c1ccccc1</smiles>

2p, $68 \%$<smiles>c1ccc(CN2c3ccccc3-c3ccccc3C2c2ccccc2)cc1</smiles>

2q, n.d.

Scheme 2. Intramolecular cyclization of biaryl tertiary amines 1a-1q promoted by $t$-BuOK/DMF.

Reaction conditions: 1a-1q $(0.2 \mathrm{mmol}), t$-BuOK $(0.6 \mathrm{mmol})$, DMF $(2.0 \mathrm{~mL})$, nitrogen atmosphere, $150{ }^{\circ} \mathrm{C}, 3 \mathrm{~h}$. Isolated yields.

With the optimal reaction conditions in hand, the reaction was extended to a variety of biaryl tertiary amines, and the results are summarized in Scheme 2. Substrates with electron-donating groups such as methoxyl and methyl groups on the aryl amine motif were well tolerated. Substrates with single and double methoxyl substitutions gave the products $\mathbf{2 b} \mathbf{2} \mathbf{2} \mathbf{e}$ in good yields. The substrates with a methyl substitution gave the products $\mathbf{2} \mathbf{f}-\mathbf{2} \mathbf{h}$ in lower yields. Radical delocalization between the methyl group and amino alkyl group accounts for the poor yield of products. The ortho-methyl substituted substrate $\mathbf{1 h}$ gave a poor yield which 
implies that this transformation is sensitive to steric hindrance. Substitution with an electron-withdrawing $\mathrm{CF}_{3}$ group showed a detrimental effect. Although complete consumption of $1 \mathbf{i}$ was observed, no expected product $\mathbf{2} \mathbf{i}$ could be isolated. A substrate with a naphthyl group was also examined and the product $\mathbf{2} \mathbf{j}$ was obtained in a moderate yield. The 2 -thienyl substituted substrate $\mathbf{1} \mathbf{k}$ was found to be unreactive.

The effect of the substitutions on the biaryl motif was also examined. The replacement of biaryl group with a naphthyl group (1) led to the loss of the reactivity. The substrates with a methyl substitution gave the products $\mathbf{2} \mathbf{m}-\mathbf{2 n}$ in poor yields. However, the substrate with a pyridyl group (10) gave a $68 \%$ yield. When the methyl group on the nitrogen was changed to ethyl, the product $2 p$ was obtained in a $68 \%$ yield. However, the benzyl replacement (1q) proved to inhibit the reaction.

To have a better understanding of the mechanism, radical trapping experiments were performed (Scheme 3). The reaction was totally inhibited in the presence of oxygen and butylated hydroxytoluene (BHT). The results implicate a radical reaction pathway.

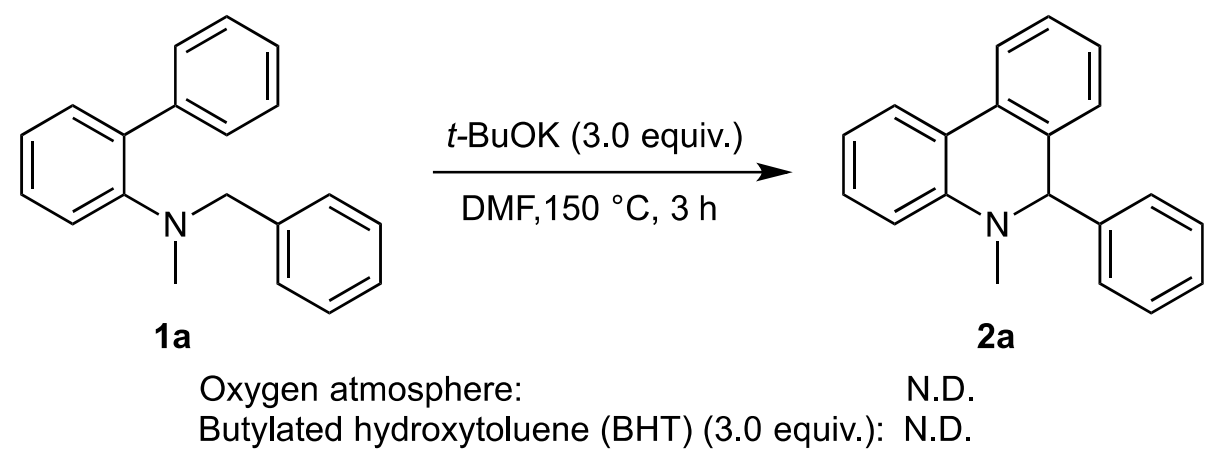

Scheme 3. Radical trapping experiments.

Based on our previous studies and the present results, a tentative reaction mechanism is proposed (Scheme 3). DMF is deprotonated by $t$-BuOK to give the carbamoyl radical A. After a single-electron transfer (SET) step, the $\alpha$-amino alkyl radical B is generated. B then undergoes an intramolecular radical addition to the phenyl ring. The resulting aryl radical $\mathbf{C}$ transfers an electron to DMF and is subsequently deprotonated by $t$-BuOK to give $\mathbf{2 a}$. 


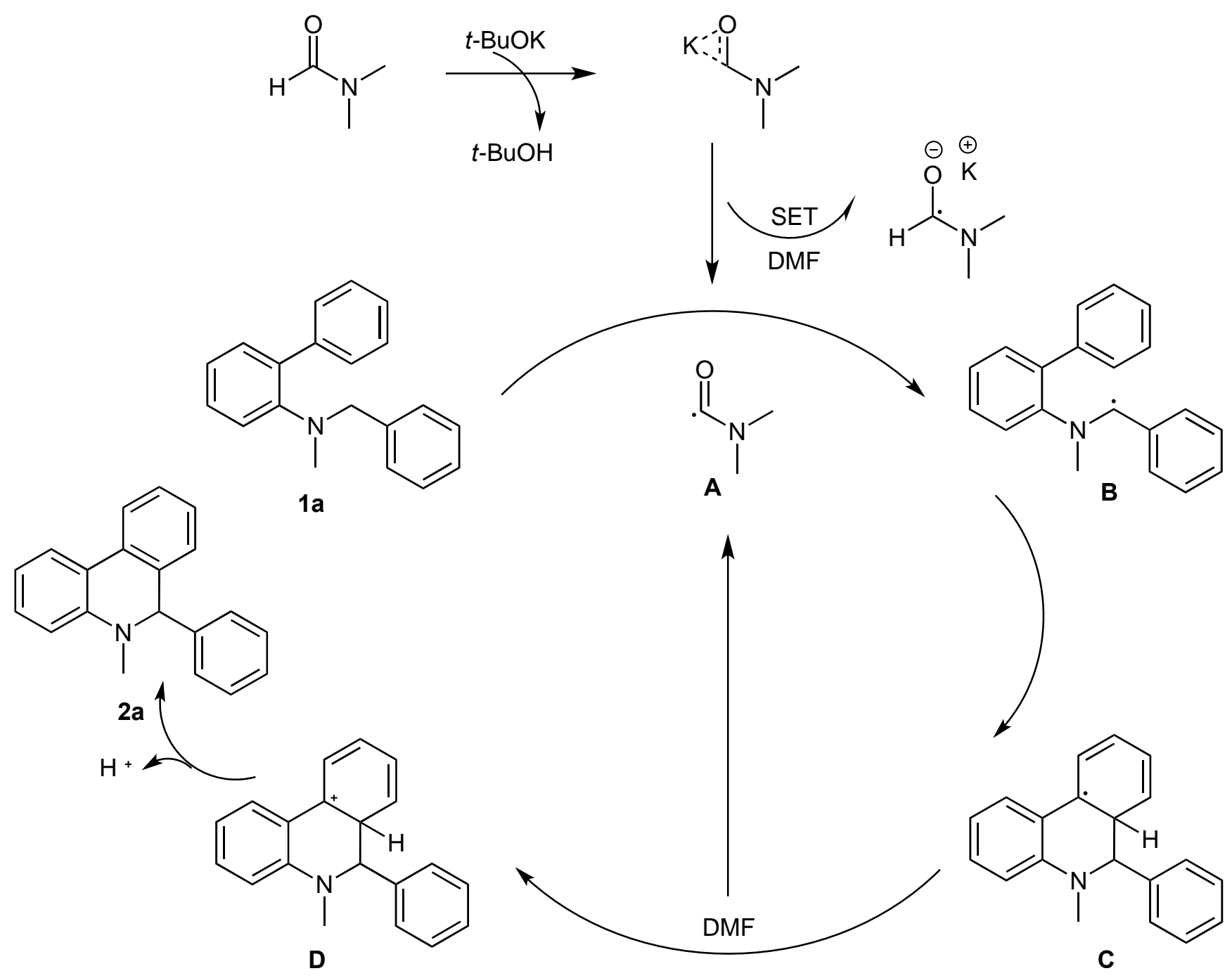

Scheme 4. Proposed reaction mechanism.

\section{Conclusions}

In summary, we have developed an intramolecular dehydrogenative coupling of biaryl tertiary amines promoted by $t$-BuOK/DMF. A number of 6 -aryl-5,6-dihydrophenanthridines were prepared in good yields. A radical reaction pathway is proposed. The finding provides a new synthetic approach to dihydrophenanthridine derivatives.

\section{Experimental Section}

General. ${ }^{1} \mathrm{H}$ NMR and ${ }^{13} \mathrm{C}$ NMR spectra were recorded on Bruker AVANCE 400 spectrometer. Chemical shifts of protons are reported in parts per million downfield from tetramethylsilane and are referenced to residual protium in the NMR solvent $\left(\mathrm{CDCl}_{3}: \delta 7.26\right)$. Chemical shifts of carbon are referenced to the carbon resonances of the solvent $\left(\mathrm{CDCl}_{3}: \delta 77.0\right)$ unless otherwise stated. Peaks are labeled as singlet $(\mathrm{s})$, broad singlet (br), doublet $(d)$, triplet $(t)$, double doublet $(d d)$, multiplet $(m)$. Melting points were measured on a WRS-2A melting 
point apparatus and are uncorrected. GC spectra were taken on an Agilent-6890A instrument. All products were further characterized by HRMS (high resolution mass spectra). The $t$-BuOK was purchased from Alfa Aesar chemical company and used without further purification. THF, DMF were dried and redistilled according to standard methods. DMSO was dried over $4 \AA \AA$ molecular sieves.

General procedure for intramolecular dehydrogenative coupling. To a dried $10 \mathrm{~mL}$ reaction tube was added 1a $(54.3 \mathrm{mg}, 0.2 \mathrm{mmol}), t$-BuOK $(67.4 \mathrm{mg}, 0.6 \mathrm{mmol})$ and DMF $(2 \mathrm{~mL})$. The mixture was stirred at $150{ }^{\circ} \mathrm{C}$ for $3 \mathrm{~h}$ under nitrogen atmosphere. Water $(20.0 \mathrm{~mL})$ was then added and the mixture was extracted with $\mathrm{CH}_{2} \mathrm{Cl}_{2}(15$ $\mathrm{mL} \times 2$ ). The combined organic layer was dried over anhydrous $\mathrm{Na}_{2} \mathrm{SO}_{4}$. After the solvent was removed, the crude product was obtained which was purification by column chromatography to give the product 2 a (36.9 $\mathrm{mg}, 68 \%)$ as a colorless oil.

5-Methyl-6-phenyl-5,6-dihydrophenanthridine (2a). ${ }^{36}$ Colorless oil. ${ }^{1} \mathrm{H}$ NMR (400 MHz, DMSO- $\left.d_{6}\right): \delta 7.54(\mathrm{~d}, J$ $7.9 \mathrm{~Hz}, 1 \mathrm{H}), 7.48$ (dd, J 7.7, $1.3 \mathrm{~Hz}, 1 \mathrm{H}), 6.99-6.94(\mathrm{~m}, 1 \mathrm{H}), 6.90-6.77(\mathrm{~m}, 8 \mathrm{H}), 6.49-6.39(\mathrm{~m}, 1 \mathrm{H}), 6.29(\mathrm{~d}, J 8.1$ $\mathrm{Hz}, 1 \mathrm{H}), 5.20(\mathrm{~s}, 1 \mathrm{H}), 3.02(\mathrm{~s}, 3 \mathrm{H}) .{ }^{13} \mathrm{C}$ NMR (100 MHz, DMSO-d $\left.)_{6}\right): \delta 149.9,146.8,140.7,135.3,134.8,133.6$, $132.8,132.6,132.6,132.2,131.6,128.29,127.5,126.5,122.6,117.4,71.0,41.9$. HRMS (ESI) calculated for $\mathrm{C}_{20} \mathrm{H}_{18} \mathrm{~N}(\mathrm{M}+\mathrm{H})^{+}:$:272.1434, found: 272.1433 .

6-(4-Methoxyphenyl)-5-methyl-5,6-dihydrophenanthridine (2b). ${ }^{36}$ White solid, M.p. $\left(153.4-156.6{ }^{\circ} \mathrm{C}\right) .{ }^{1} \mathrm{H}$ NMR $\left(400 \mathrm{MHz}, \mathrm{CDCl}_{3}\right): \delta 7.81(\mathrm{~d}, J 7.5 \mathrm{~Hz}, 1 \mathrm{H}), 7.76(\mathrm{dd}, J 7.7,1.4 \mathrm{~Hz}, 1 \mathrm{H}), 7.28(\mathrm{td}, J 7.7,1.4 \mathrm{~Hz}, 1 \mathrm{H}), 7.22-$ $7.15(\mathrm{~m}, 2 \mathrm{H}), 7.06-7.02(\mathrm{~m}, 3 \mathrm{H}), 6.83(\mathrm{td}, J \mathrm{7} .5,1.0 \mathrm{~Hz}, 1 \mathrm{H}), 6.71-6.67(\mathrm{~m}, 2 \mathrm{H}), 6.59(\mathrm{~d}, J 8.1 \mathrm{~Hz}, 1 \mathrm{H}), 5.31(\mathrm{~s}$, 1H), 3.69 (s, 3H), 2.87 (s, 3H). ${ }^{13} \mathrm{C} N M R\left(100 \mathrm{MHz}, \mathrm{CDCl}_{3}\right): \delta 159.0,144.8,136.0,133.9,130.6,129.4,128.0$, $127.5,127.3,127.0,123.1,122.5,122.0,117.7,113.8,112.5,67.2,55.1,37.0$. HRMS (ESI) calculated for $\mathrm{C}_{21} \mathrm{H}_{20} \mathrm{NO}(\mathrm{M}+\mathrm{H})^{+}: 302.1539$, found: 302.1554 .

6-(3-Methoxyphenyl)-5-methyl-5,6-dihydrophenanthridine (2c). ${ }^{36}$ White solid, M.p. $\left(133.4-146.2{ }^{\circ} \mathrm{C}\right) .{ }^{1} \mathrm{H}$ NMR $\left(400 \mathrm{MHz}, \mathrm{CDCl}_{3}\right): \delta 7.80(\mathrm{~d}, J 7.5 \mathrm{~Hz}, 1 \mathrm{H}), 7.75(\mathrm{dd}, J$ 7.7, $1.4 \mathrm{~Hz}, 1 \mathrm{H}), 7.30-7.25(\mathrm{~m}, 1 \mathrm{H}), 7.22-7.14(\mathrm{~m}$, $2 \mathrm{H}), 7.10-7.05(\mathrm{~m}, 2 \mathrm{H}), 6.82(\mathrm{td}, J 7.5,1.1 \mathrm{~Hz}, 1 \mathrm{H}), 6.73(\mathrm{dd}, J 7.7,1.1 \mathrm{~Hz}, 1 \mathrm{H}), 6.71-6.66(\mathrm{~m}, 2 \mathrm{H}), 6.61(\mathrm{~d}, J 8.2$ $\mathrm{Hz}, 1 \mathrm{H}), 5.32(\mathrm{~s}, 1 \mathrm{H}), 3.60(\mathrm{~s}, 3 \mathrm{H}), 2.88(\mathrm{~s}, 3 \mathrm{H}) .{ }^{13} \mathrm{C} \mathrm{NMR}\left(100 \mathrm{MHz}_{\mathrm{CDCl}}\right): \delta 159.6,145.0,143.2,135.6,130.65$, $129.5,127.7,127.3,127.0,123.2,122.5,122.0,119.1,117.8,112.8,112.5,112.4,67.9,55.0,37.2$. HRMS (ESI) calculated for $\mathrm{C}_{21} \mathrm{H}_{20} \mathrm{NO}(\mathrm{M}+\mathrm{H})^{+}: 302.1539$, found: 302.1550 .

6-(3,4-Dimethoxyphenyl)-5-methyl-5,6-dihydrophenanthridine (2d). Colorless oil. ${ }^{1} \mathrm{H} \mathrm{NMR}\left(400 \mathrm{MHz}^{\mathrm{CDCl}} 3\right)$ : $\delta 7.81(\mathrm{~d}, J 7.9 \mathrm{~Hz}, 1 \mathrm{H}), 7.76(\mathrm{dd}, J 7.7,1.5 \mathrm{~Hz}, 1 \mathrm{H}), 7.29(\mathrm{td}, J$ 7.7, $1.4 \mathrm{~Hz}, 1 \mathrm{H}), 7.23-7.20(\mathrm{~m}, 1 \mathrm{H}), 7.19-7.16(\mathrm{~m}$, $1 \mathrm{H}), 7.09-7.06(\mathrm{~m}, 1 \mathrm{H}), 6.83(\mathrm{td}, J 7.5,1.1 \mathrm{~Hz}, 1 \mathrm{H}), 6.71(\mathrm{dd}, J$ 8.2, $1.9 \mathrm{~Hz}, 1 \mathrm{H}), 6.69-6.66(\mathrm{~m}, 1 \mathrm{H}), 6.63(\mathrm{~d}, J 1.6$ $\mathrm{Hz}, 1 \mathrm{H}), 6.61(\mathrm{~d}, J 0.6 \mathrm{~Hz}, 1 \mathrm{H}), 5.27(\mathrm{~s}, 1 \mathrm{H}), 3.77(\mathrm{~s}, 3 \mathrm{H}), 3.58(\mathrm{~s}, 3 \mathrm{H}), 2.88(\mathrm{~s}, 3 \mathrm{H}) .{ }^{13} \mathrm{C} \mathrm{NMR}\left(100 \mathrm{MHz}, \mathrm{CDCl}_{3}\right): \delta$ $148.9,148.6,145.1,136.2,134.3,130.6,129.5,127.6,127.3,126.9,123.1,122.5,122.3,119.0,117.8,112.6$, 111.0, 109.9, 67.6, 55.8, 55.6, 37.1. HRMS (ESI) calculated for $\mathrm{C}_{22} \mathrm{H}_{22} \mathrm{NO}_{2}(\mathrm{M}+\mathrm{H})^{+}:$332.1645, found: 332.1643.

6-(2,5-Dimethoxyphenyl)-5-methyl-5,6-dihydrophenanthridine (2e). Colorless oil. $\left.{ }^{1} \mathrm{H} \mathrm{NMR} \mathrm{(400} \mathrm{MHz}_{1} \mathrm{CDCl}_{3}\right)$ : $\delta 7.78(\mathrm{~d}, J 7.8 \mathrm{~Hz}, 1 \mathrm{H}), 7.74(\mathrm{dd}, J 7.7,1.4 \mathrm{~Hz}, 1 \mathrm{H}), 7.19(\mathrm{~m}, 4 \mathrm{H}), 6.83-6.80(\mathrm{~m}, 1 \mathrm{H}), 6.78(\mathrm{~s}, 1 \mathrm{H}), 6.73-6.64(\mathrm{~m}$, $1 \mathrm{H}), 6.62(\mathrm{~m}, 1 \mathrm{H}), 6.57(\mathrm{~d}, J 3.1 \mathrm{~Hz}, 1 \mathrm{H}), 6.05(\mathrm{~s}, 1 \mathrm{H}), 3.86(\mathrm{~s}, 3 \mathrm{H}), 3.40(\mathrm{~s}, 3 \mathrm{H}), 2.87(\mathrm{~s}, 3 \mathrm{H}) .{ }^{13} \mathrm{C} \mathrm{NMR}(100 \mathrm{MHz}$, $\left.\mathrm{CDCl}_{3}\right): \delta 153.6,149.7,145.5,136.2,131.5,130.8,129.4,127.4,127.2,126.8,123.1,122.4,122.0,117.5,113.4$, 113.4, 112.1, 111.9, 58.9, 56.2, 55.2, 36.8. HRMS (ESI) calculated for $\mathrm{C}_{22} \mathrm{H}_{22} \mathrm{NO}_{2}(\mathrm{M}+\mathrm{H})^{+}: 332.1645$, found: 332.1639.

5-Methyl-6-p-tolyl-5,6-dihydrophenanthridine (2f). ${ }^{36}$ Colorless oil. ${ }^{1} \mathrm{H}$ NMR $\left(400 \mathrm{MHz}, \mathrm{CDCl}_{3}\right): \delta 7.81(\mathrm{t}, J 6.5$ $\mathrm{Hz}, 1 \mathrm{H}), 7.75(\mathrm{dd}, J$ 7.7, $1.4 \mathrm{~Hz}, 1 \mathrm{H}), 7.29-7.25(\mathrm{~m}, 1 \mathrm{H}), 7.23-7.18(\mathrm{~m}, 1 \mathrm{H}), 7.18-7.14(\mathrm{~m}, 1 \mathrm{H}), 7.07-7.04(\mathrm{~m}$, $1 \mathrm{H}), 7.02(\mathrm{dd}, J 6.6,4.8 \mathrm{~Hz}, 2 \mathrm{H}), 6.96(\mathrm{~d}, J 8.1 \mathrm{~Hz}, 2 \mathrm{H}), 6.84-6.80(\mathrm{~m}, 1 \mathrm{H}), 6.59(\mathrm{~d}, J 8.0 \mathrm{~Hz}, 1 \mathrm{H}), 5.32(\mathrm{~s}, 1 \mathrm{H})$, $2.87(\mathrm{~s}, 3 \mathrm{H}), 2.22(\mathrm{~s}, 3 \mathrm{H}) .{ }^{13} \mathrm{C} \mathrm{NMR}\left(100 \mathrm{MHz}, \mathrm{CDCl}_{3}\right): \delta 144.9,138.6,137.2,135.9,130.7,129.4,129.2,128.3$, 
127.5, 127.3, 127.0, 126.7, 123.1, 122.5, 117.6, 112.4, 67.6, 37.1, 21.0. HRMS (ESI) calculated for $\mathrm{C}_{21} \mathrm{H}_{20} \mathrm{~N}$ $(\mathrm{M}+\mathrm{H})^{+}:$286.1590, found: 286.1603 .

5-Methyl-6-m-tolyl-5,6-dihydrophenanthridine (2g). Colorless oil. ${ }^{1} \mathrm{H} \mathrm{NMR}\left(400 \mathrm{MHz}, \mathrm{CDCl}_{3}\right): \delta 7.83-7.72(\mathrm{~m}$, $2 \mathrm{H}), 7.27(\mathrm{~d}, J 7.3 \mathrm{~Hz}, 1 \mathrm{H}), 7.19(\mathrm{~m}, 2 \mathrm{H}), 7.04(\mathrm{~m}, 2 \mathrm{H}), 6.98-6.89(\mathrm{~m}, 3 \mathrm{H}), 6.83(\mathrm{dd}, J 11.8,7.7 \mathrm{~Hz}, 1 \mathrm{H}), 6.63-6.57$ $(\mathrm{m}, 1 \mathrm{H}), 5.32(\mathrm{~s}, 1 \mathrm{H}), 2.87(\mathrm{~s}, 3 \mathrm{H}), 2.21(\mathrm{~s}, 3 \mathrm{H}) .{ }^{13} \mathrm{C} \mathrm{NMR}\left(100 \mathrm{MHz}, \mathrm{CDCl}_{3}\right): \delta 145.0,141.6,138.0,135.8,130.6$, 129.5, 128.5, 128.4, 127.6, 127.5, 127.3, 127.1, 123.9, 123.2, 122.5, 121.9, 117.6, 112.4, 67.9, 37.2, 21.6. HRMS (ESI) calculated for $\mathrm{C}_{21} \mathrm{H}_{20} \mathrm{~N}(\mathrm{M}+\mathrm{H})^{+}:$:286.1590, found: 286.1585 .

5-Methyl-6-o-tolyl-5,6-dihydrophenanthridine (2h). Colorless oil. ${ }^{1} \mathrm{H}$ NMR $\left(400 \mathrm{MHz}, \mathrm{CDCl}_{3}\right): \delta 7.81$ (d, J 7.5 $\mathrm{Hz}, 1 \mathrm{H}), 7.76(\mathrm{dd}, J$ 7.7, $1.4 \mathrm{~Hz}, 1 \mathrm{H}), 7.23-7.20(\mathrm{~m}, 1 \mathrm{H}), 7.20-7.16(\mathrm{~m}, 1 \mathrm{H}), 7.16-7.11(\mathrm{~m}, 2 \mathrm{H}), 7.11-7.06(\mathrm{~m}$, $2 \mathrm{H}$ ), $7.00(\mathrm{dd}, J 7.2,6.0 \mathrm{~Hz}, 1 \mathrm{H}), 6.81(\mathrm{dd}, J 10.7,4.3 \mathrm{~Hz}, 2 \mathrm{H}), 6.62(\mathrm{~d}, J 7.9 \mathrm{~Hz}, 1 \mathrm{H}), 5.82(\mathrm{~s}, 1 \mathrm{H}), 2.73(\mathrm{~s}, 3 \mathrm{H})$, 2.48 (s, 3H). ${ }^{13} \mathrm{C}$ NMR $\left(100 \mathrm{MHz}, \mathrm{CDCl}_{3}\right): \delta$ 145.6, 141.0, 136.1, 134.7, 130.9, 130.8, 129.5, 128.8, 127.5, 127.4, $127.2,126.8,126.6,123.1,122.1,121.4,117.5,112.1,63.5,36.3,20.1$. HRMS (ESI) calculated for $\mathrm{C}_{21} \mathrm{H}_{20} \mathrm{~N}$ $(\mathrm{M}+\mathrm{H})^{+}: 286.1590$, found: 286.1580 .

5-Methyl-6-(naphthalen-2-yl)-5,6-dihydrophenanthridine (2j). Colorless oil. ${ }^{1} \mathrm{H} N M R\left(400 \mathrm{MHz}, \mathrm{CDCl}_{3}\right): \delta 7.84$ (d, J 7.8 Hz, 1H), $7.79(\mathrm{dd}, J$ 7.7, $1.4 \mathrm{~Hz}, 1 \mathrm{H}), 7.75-7.71(\mathrm{~m}, 1 \mathrm{H}), 7.71-7.68(\mathrm{~m}, 1 \mathrm{H}), 7.63-7.58(\mathrm{~m}, 2 \mathrm{H}), 7.39(\mathrm{~m}$, 2H), 7.31-7.26 (m, 1H), 7.24-7.19 (m, 2H), 7.17-7.13 (m, 1H), 7.08 (dd, J 7.6, $1.1 \mathrm{~Hz}, 1 \mathrm{H}), 6.85(\mathrm{td}, J 7.6,1.0 \mathrm{~Hz}$, $1 \mathrm{H}), 6.61(\mathrm{~d}, J 8.0 \mathrm{~Hz}, 1 \mathrm{H}), 5.52(\mathrm{~s}, 1 \mathrm{H}), 2.89(\mathrm{~s}, 3 \mathrm{H}) .{ }^{13} \mathrm{C} \mathrm{NMR}\left(100 \mathrm{MHz}, \mathrm{CDCl}_{3}\right): \delta$ 145.0, 139.3, 135.5, 133.2, 133.0, 130.8, 129.6, 128.6, 128.1, 127.7, 127.6, 127.3, 126.1, 125.8, 125.2, 125.2, 123.2, 122.5, 121.82, 117.8, 112.4, 68.1, 37.1. HRMS (ESI) calculated for $\mathrm{C}_{24} \mathrm{H}_{20} \mathrm{~N}(\mathrm{M}+\mathrm{H})^{+}: 322.1590$, found: 322.1592 .

5,10-Dimethyl-6-phenyl-5,6-dihydrophenanthridine (2m). Colorless oil. ${ }^{1} \mathrm{H} N M R\left(400 \mathrm{MHz}, \mathrm{CDCl}_{3}\right): \delta 7.81(\mathrm{~d}, J$ $7.9 \mathrm{~Hz}, 1 \mathrm{H}), 7.77(\mathrm{~d}, J 7.7 \mathrm{~Hz}, 1 \mathrm{H}), 7.24-7.13(\mathrm{~m}, 4 \mathrm{H}), 7.12-7.07(\mathrm{~m}, 2 \mathrm{H}), 7.00(\mathrm{t}, J 7.2 \mathrm{~Hz}, 1 \mathrm{H}), 6.83(\mathrm{t}, J 7.6 \mathrm{~Hz}$, $2 \mathrm{H}), 6.62(\mathrm{~d}, J 8.2 \mathrm{~Hz}, 1 \mathrm{H}), 5.82(\mathrm{~s}, 1 \mathrm{H}), 2.74(\mathrm{~s}, 3 \mathrm{H}), 2.49(\mathrm{~s}, 3 \mathrm{H}) .{ }^{13} \mathrm{C} \mathrm{NMR}\left(100 \mathrm{MHz}, \mathrm{CDCl}_{3}\right): \delta$ 145.6, 141.0, $136.1,134.7,131.8,130.9,129.5,128.7,127.5,127.5,127.2,126.8,126.6,123.1,122.2,121.4,117.5,112.1$, 63.4, 36.3, 20.2. HRMS (ESI) calculated for $\mathrm{C}_{21} \mathrm{H}_{20} \mathrm{~N}(\mathrm{M}+\mathrm{H})^{+}: 286.1590$, found: 286.1580 .

5,8-Dimethyl-6-phenyl-5,6-dihydrophenanthridine (2n). Colorless oil. ${ }^{1} \mathrm{H} N M R\left(400 \mathrm{MHz}, \mathrm{CDCl}_{3}\right): \delta 7.75-7.68$ $(\mathrm{m}, 2 \mathrm{H}), 7.20-7.14(\mathrm{~m}, 4 \mathrm{H}), 7.13-7.08(\mathrm{~m}, 3 \mathrm{H}), 6.88(\mathrm{~s}, 1 \mathrm{H}), 6.82(\mathrm{td}, J 7.5,1.0 \mathrm{~Hz}, 1 \mathrm{H}), 6.57(\mathrm{~d}, J 8.0 \mathrm{~Hz}, 1 \mathrm{H})$, $5.30(\mathrm{~s}, 1 \mathrm{H}), 2.86(\mathrm{~s}, 3 \mathrm{H}), 2.27(\mathrm{~s}, 3 \mathrm{H}) .{ }^{13} \mathrm{C} \mathrm{NMR}\left(100 \mathrm{MHz}, \mathrm{CDCl}_{3}\right): \delta 144.6,141.7,137.1,135.6,129.0,128.6$, $128.5,128.0,127.6,127.6,126.8,122.8,122.5,122.1,117.6,112.3,68.0,37.1,21.2$. HRMS (ESI) calculated for $\mathrm{C}_{21} \mathrm{H}_{20} \mathrm{~N}(\mathrm{M}+\mathrm{H})^{+}:$:286.1590, found: 286.1583 .

6-Methyl-5-phenyl-5,6-dihydrobenzo[c][2,7]naphthyridine (2o). Yellow oil. ${ }^{1} \mathrm{H} \mathrm{NMR}\left(400 \mathrm{MHz}, \mathrm{CDCl}_{3}\right): \delta 8.45$ (d, J $5.4 \mathrm{~Hz}, 1 \mathrm{H}), 8.32(\mathrm{~s}, 1 \mathrm{H}), 7.75(\mathrm{dd}, J 7.8,1.3 \mathrm{~Hz}, 1 \mathrm{H}), 7.58(\mathrm{~d}, J 5.3 \mathrm{~Hz}, 1 \mathrm{H}), 7.33-7.28(\mathrm{~m}, 1 \mathrm{H}), 7.19(\mathrm{dd}, J$ 6.3, 3.6 Hz, 3H), 7.14 (dd, J 6.6, 3.1 Hz, 2H), $6.84(\mathrm{dd}, J$ 10.9, $4.1 \mathrm{~Hz}, 1 \mathrm{H}), 6.65(\mathrm{~d}, J 8.2 \mathrm{~Hz}, 1 \mathrm{H}), 5.45(\mathrm{~s}, 1 \mathrm{H}), 2.92$ (s, 3H). ${ }^{13} \mathrm{C} \mathrm{NMR}\left(100 \mathrm{MHz}, \mathrm{CDCl}_{3}\right): \delta 148.6,148.4,146.0,141.2,137.9,131.9,130.1,128.8,128.0,126.4,124.1$, 118.8, 117.7, 116.0, 112.6, 65.1, 37.2. HRMS (ESI) calculated for $\mathrm{C}_{19} \mathrm{H}_{17} \mathrm{~N}_{2}(\mathrm{M}+\mathrm{H})^{+}:$: 273.1386, found: 273.1364.

5-Ethyl-6-phenyl-5,6-dihydrophenanthridine (2p). Yellow oil. ${ }^{1} \mathrm{H} N M R\left(400 \mathrm{MHz}, \mathrm{CDCl}_{3}\right): \delta 7.81-7.71(\mathrm{~m}, 2 \mathrm{H})$, 7.27-7.23 (m, 1H), 7.21-7.17 (m, 3H), 7.14 (m, 3H), 7.10-7.01 (m, 2H), $6.79(\mathrm{~m}, 1 \mathrm{H}), 6.72(\mathrm{~d}, J 8.2 \mathrm{~Hz}, 1 \mathrm{H}), 5.49$ $(\mathrm{s}, 1 \mathrm{H}), 3.47(\mathrm{~m}, 1 \mathrm{H}), 3.25(\mathrm{~m}, 1 \mathrm{H}), 1.17(\mathrm{t}, J 7.1 \mathrm{~Hz}, 3 \mathrm{H}) .{ }^{13} \mathrm{C} \mathrm{NMR}\left(100 \mathrm{MHz}, \mathrm{CDCl}_{3}\right): \delta 144.2,143.6,135.7$, $130.6,129.4,128.5,127.5,127.4,127.1,127.0,126.6,123.5,122.5,121.7,117.2,112.4,65.5,44.1,12.6$. HRMS (ESI) calculated for $\mathrm{C}_{21} \mathrm{H}_{20} \mathrm{~N}(\mathrm{M}+\mathrm{H})^{+}: 286.1517286 .1590$, found: 286.1611 . 


\section{Acknowledgements}

We thank the National Natural Science Foundation of China (No. 21472248), the Guangdong Engineering Research Center of Chiral Drugs and the Innovative Scientific Research Team Introducing Project of Zhongshan City (No. 2015-224) for the financial support of this study.

\section{References}

1. Girard, S. A.; Knauber, T.; Li, C. J. Angew. Chem. Int. Ed. 2014, 53, 74. https://doi.org/10.1002/anie.201304268

2. Shi, Z.; Glorius F. Angew. Chem. Int. Ed. 2012, 51, 9220. https://doi.org/10.1002/anie.201205079

3. Yeung, C. S.; Dong, V. M. Chem. Rev. 2011, 111, 1215. https://doi.org/10.1021/cr100280d

4. Li, C. J. Acc. Chem. Res. 2009, 42, 335. https://doi.org/10.1021/ar800164n

5. Wu, C. J.; Meng, Q. Y.; Lei, T.; Zhong, J. J.; Liu, W. Q.; Zhao, L. M.; Li, Z. J.; Chen, B.; Tung, C. H.; Wu. L. Z. ACS Catal. 2016, 6, 4635. https://doi.org/10.1021/acscatal.6b00917

6. Mei, S. T.; Liang, H. W.; Teng, B.; Wang, N. J.; Shuai. L.; Yuan, Y.; Chen, Y. C.; Wei, Y. Org. Lett. 2016, $18,1088$. https://doi.org/10.1021/acs.orglett.6b00197

7. Cambeiro, X. C.; Ahlsten, N.; Larrosa, I. J. Am. Chem. Soc. 2015, 137,15636. https://doi.org/10.1021/jacs.5b10593

8. Wang, J.; Liu, C.; Yuan, J. W.; Lei, A. W. Angew. Chem. Int. Ed. 2013, 52, 2256. https://doi.org/10.1002/anie.201208920

9. Kuhl, N.; Hopkinson, M. N.; Glorius, F. Angew. Chem. Int. Ed. 2012, 51, 8230. https://doi.org/10.1002/anie.201203792

10. Zhang, G.; Zhang, Y. H.; Wang, R. Angew. Chem. Int. Ed. 2011, 50, 10429. https://doi.org/10.1002/anie.201105123

11. Volvoikar, P. S.; Tilve, S. G. Org. Lett. 2016, 18, 892. https://doi.org/10.1021/acs.orglett.5b03392

12. Martínez, C.; Bosnidou, A. E.; Allmendinger, S.; Muñiz, K. Chem. Eur. J. 2016, 22, 1. https://doi.org/10.1002/chem.201504553

13. Liu, J.; Zhang, H.; Yi, H.; Liu, C.; Lei, A. W. Sci. China Chem. 2015, 58, 1323. https://doi.org/10.1007/s11426-015-5381-2

14. Laha, J. K.; Tummalapalli, K. S. S.; Nair, A.; Patel, N. J. Org. Chem. 2015, 80, 11351 https://doi.org/10.1021/acs.joc.5b01872

15. Sun, X.; Hu, Y.; Nie, S. Z.; Yan, Y. Y.; Zhang, X. J.; Yan, M. Adv. Synth. Catal. 2013, 355, 2179 https://doi.org/10.1002/adsc.201300455

16. Kim, H. J.; Kim, J. Y.; Cho, S. H.; Chang, S. J. Am. Chem. Soc. 2011, 133, 16382. https://doi.org/10.1021/ja207296y

17. Chen, X. P.; Cui, X. L.; Yang, F. F.; Wu, Y. J. Org. Lett. 2015, 17, 1445. https://doi.org/10.1021/acs.orglett.5b00330 
18. Kumar, S.; Rathore, V.; Verma, A.; Prasaad, C. D.; Kumar, A.; Yadav, A.; Jana, S.; Sattar, M.; Meenakshi, S.; Kumar, S. Org. Lett. 2015, 17, 82.

https://doi.org/10.1021/ol503274z

19. Chen, Y. Y.; Chen, J. H.; Zhang, N. N.; Ye, L. M.; Zhang X. J.; Yan, M. Tetrahedron Lett. 2015, 56, 478.

https://doi.org/10.1016/j.tetlet.2014.12.002

20. Wei, W. T.; Liu, Y.; Ye, L. M.; Lei, R. H.; Zhang X. J.; Yan, M. Org. Biomol. Chem. 2015, 13, 817. https://doi.org/10.1039/C4OB01948B

21. Chen, Y. Y.; Zhang, X. J.; Yuan, H. M.; Wei, W. T.; Yan, M. Chem. Commun. 2013, 49, 10974. https://doi.org/10.1039/c3cc46340k

22. Wei, W. T.; Dong, X. J.; Nie, S. Z.; Chen, Y. Y.; Zhang, X. J.; Yan, M. Org. Lett. 2013, 15, 6018. https://doi.org/10.1021/ol402908m

23. Wang, W. J.; Zhao, X.; Tong, L.; Chen, J. H.; Zhang, X. J.; Yan, M. J. Org. Chem. 2014, 79, 8557. https://doi.org/10.1021/j0501179t

24. Chen, Y. Y.; Chen, Z. Y.; Zhang, N. N.; Chen, J. H.; Zhang X. J.; Yan, M. Eur. J. Org. Chem. 2016, 2016, 599.

25. Chen, Y. Y.; Zhang, N. N.; Ye, L. M.; Chen, J. H.; Sun, X.; Zhang X. J.; Yan, M. RSC Adv. 2015, 5, 48046. https://doi.org/10.1039/C5RA07188G

26. Pegoraro, S.; Lang, M.; Dreker, T.; Kraus, J.; Hamm, S.; Meere, C.; Feurle, J.; Tasler, S.; Pruetting, S.; Kuras, Z.; Visan, V.; Grissmer, S. Bioorg. Med. Chem. Lett. 2011, 21, 5647.

https://doi.org/10.1016/j.bmcl.2011.07.013

27. Miao, F.; Yang, X.-J.; Zhou, L.; Hu, H.-J.; Zheng, F.; Ding, X.-D.; Sun, D.-M.; Zhou, C.-D.; Sun, W. Nat. Prod. Res. 2011, $25,863$. https://doi.org/10.1080/14786419.2010.482055

28. Fotie, J.; Bohle, D. S.; Olivier, M.; Gomez, M. A.; Nzimiro, S. J. Nat. Prod. 2007, 70, 1650. https://doi.org/10.1021/np0702281

29. Kundu, K.; Knight, S. F.; Lee, S.; Taylor, W. R.; Murthy N. Angew. Chem. Int. Ed. 2010, 49, 6134. https://doi.org/10.1002/anie.201002228

30. Ye, J.; Limouni, A.; Zaichuk, S.; Lautens M. Angew. Chem. Int. Ed. 2015, 54, 3116. https://doi.org/10.1002/anie.201411276

31. Augustine, J. K.; Bombrun, A.; Alagarsamy, P.; Jothi, A. Tetrahedron Lett. 2012, 53, 6280. https://doi.org/10.1016/j.tetlet.2012.09.034

32. Meseroll, L. M. N.; McKee, J. R.; Zanger, M. Synth. Commun. 2011, 41, 2557. https://doi.org/10.1080/00397911.2010.515329

33. Mandadapu, A. K.; Saifuddin, M.; Agarwal, P. K.; Kundu, B. Org. Biomol. Chem. 2009, 7, 2796. https://doi.org/10.1039/b905696c

34. Pegoraro, S.; Lang, M.; Dreker, T.; Kraus, J.; Hamm, S.; Meere, C.; Feurle, J.; Tasler, S.; Pruetting, S.; Kuras, Z.; Visan, V.; Grissmer, S. Bioorg. Med. Chem. Lett. 2009, 19, 2299.

https://doi.org/10.1016/j.bmcl.2009.02.077

35. Castillo, J. C.; Quiroga, J.; Abonia, R.; Rodriguez, J.; Coquerel, Y. J. Org. Chem. 2015, 80, 9767. https://doi.org/10.1021/acs.joc.5b01564

36. Zhu, X. Q.; Li, X. T.; Han, S. H.; Mei L. R. J. Org. Chem. 2012, 77, 4774. https://doi.org/10.1021/jo3005952 